\title{
Hybrid quantum-classical circuit simplification with the ZX-calculus
}

\author{
Agustín Borgna $^{1,2}[0000-0002-1688-1370]$, Simon Perdrix [0000-0002-1808-2409], \\ and Benoît Valiron 3 [0000-0002-1008-5605] \\ 1 CNRS LORIA, Inria-MOCQUA, Université de Lorraine, F 54000 Nancy, France \\ 2 Université Paris-Saclay, CNRS, Laboratoire Méthodes Formelles, 91405, Orsay, \\ France \\ 3 École CentraleSupélec, Laboratoire Méthodes Formelles, 91405, Orsay, France
}

\begin{abstract}
We present a complete optimization procedure for hybrid quantum-classical circuits with classical parity logic. While common optimization techniques for quantum algorithms focus on rewriting solely the pure quantum segments, there is interest in applying a global optimization process for applications such as quantum error correction and quantum assertions. This work, based on the pure-quantum circuit optimization procedure by Duncan et al., uses an extension of the formal graphical ZX-calculus called $\mathrm{ZX}_{\doteq}$ as an intermediary representation of the hybrid circuits to allow for granular optimizations below the quantum-gate level. We define a translation from hybrid circuits into diagrams that admit the graph-theoretical focused-gFlow property, needed for the final extraction back into a circuit. We then derive a number of gFlow-preserving optimization rules for $\mathrm{ZX}_{\perp}$ diagrams that reduce the size of the graph, and devise a strategy to find optimization opportunities by rewriting the diagram guided by a Gauss elimination process. Then, after extracting the circuit, we present a general procedure for detecting segments of circuit-like $\mathrm{ZX}_{\perp}$ - diagrams which can be implemented with classical gates in the extracted circuit. We have implemented our optimization procedure as an extension to the open-source python library PyZX.
\end{abstract}

Keywords: ZX-calculus $\cdot$ Optimization $\cdot$ Gflow $\cdot$ Hybrid circuits $\cdot$ PyZX

\section{Introduction}

The description of quantum algorithms commonly involves quantum operations interacting with classical data in its inputs, outputs, or intermediary steps via measurements or state preparations. Some applications such as quantum error correction [9, 22 and quantum assertions [18, 25] explicitly introduce classical measurements and logic between quantum computations. In general, quantum programming languages usually allow for measurements and classically controlled quantum operators mixed-in with unitary gates [11, 7, 15, 23. Furthermore, Jozsa [14 conjectured that any polynomial-time quantum algorithm can be simulated by polylogarithmic-depth quantum computation interleaved with 
polynomial-depth classical computation. As such, there is interest in contemplating this kind of structures in circuits.

A popular alternative representation of quantum circuit is based on the $Z X$ calculus [5, 6, a formal diagrammatic language which presents a more granular representation of quantum circuits and has been successfully used in applications such as MBQC [10], quantum error correction [4], and quantum foundations. Carette et al. [3] introduced an extension of the calculus called $\mathrm{ZX}_{\doteq}$ which allows for the representation of operations interacting with the classical environment by adding a discarding ground generator to the diagrams.

It is natural to look at the problem of optimizing algorithm implementations by taking in consideration the environment in addition to the pure quantum fragments. However, most common optimization strategies focus solely on the latter without contemplating the hybrid quantum-classical structure [1, 12. One of this pure optimizations introduced by Duncan et al. [8] uses the ZX-calculus to apply granular rewriting rules that ignore the boundaries of each quantum gate. We will refer to it as the Clifford optimization algorithm. Their rewriting steps preserve a diagram property called gFlow admittance that is required for the final extraction of the ZX diagrams into circuits. The ZX optimization method was latter used by Kissinger and van de Wetering [17] in their method to reduce the number of T-gates in quantum circuits.

In this work we define the natural extension of the pure Clifford optimization algorithm by Duncan et al. to hybrid quantum-classical circuits using the $\mathrm{ZX}_{\doteq}$ calculus.

Our circuit optimization procedure forgets the difference between quantum and classical wires during the simplification process, representing connections as a single type of edge. This allows it to optimize the complete hybrid system as an homogeneous diagram, and results in similar representations for operations that can be done either quantumly or classically. Generally, in a physical quantum computer, the classical operations are simpler to implement than their quantum counterparts, and quantum simulators can exploit the knowledge of which wires carry classical data to simplify their operation. As such, it is beneficial to extract classical gates in the resulting circuit where possible.

The contribution of this paper are as follows.

- We specify a translation of hybrid circuits into $\mathrm{ZX}_{\stackrel{\bar{\tau}}{\perp}}$ diagrams in a special graph like form that admits a gFlow, restricting the classical segments of the input to parity circuits.

- We introduce a number of gFlow-preserving rewriting rules that interact with the discarding generator to reduce the size of the diagrams, and devise a strategy to find optimization opportunities using the biadjacency matrix of the graph cut between spiders connected to ground generators and the other nodes in the diagram.

- We define a procedure to extract $\mathrm{ZX}_{\doteq}$ diagrams with a gFlow back into hybrid quantum-classical circuits, including ancilla initialization and termination. 
- We define the problem of $\mathrm{ZX}_{\lrcorner}$-classicalization as labelling segments of the diagrams which can be implemented classically and present an heuristic solution. Our method can be applied on the extracted circuits to maximize the number of classically implemented operations.

The paper is organized in the following manner. In Section 2 we define the quantum circuits, present a syntactic description of the $\mathrm{ZX}_{\doteq}$ calculus and its equation, and give an intuition behind the representation of hybrid quantumclassical circuits. Section 3 then introduces the graph-like family of $\mathrm{ZX}_{\doteq}$ diagrams and defines the focused gFlow property over the graphs. We then define the translation of quantum circuits into graph-like diagrams in Section 4 . In Section 5 we introduce the optimization rules and our strategy for finding rule matches which we use to describe the complete optimization algorithm. Then in Section 6 we define the extraction algorithm and finally we present our classicalization procedure in Section 7. In Section 8 we discuss the results of testing our procedure on randomly generated circuits.

\section{Hybrid quantum-classical circuits and the grounded ZX-calculus}

In pure quantum operations, a single qubit quantum state is represented as a unitary vector in the Hilbert space $\mathbb{C}^{2}$. We use Dirac notation to talk about such vectors and denote an arbitrary state as $|\phi\rangle$. States can be be described as a linear combination of vectors in a basis such as the computational basis $\{|0\rangle,|1\rangle\}$ or the diagonal basis $\{|+\rangle,|-\rangle\}$, where $| \pm\rangle=\frac{1}{\sqrt{2}}(|0\rangle \pm|1\rangle)$. A third, less commonly used basis called $Y$ is formed by the vectors $|\circlearrowright\rangle=\frac{1}{\sqrt{2}}(|0\rangle+i|1\rangle)$ and $|\circlearrowleft\rangle=\frac{1}{\sqrt{2}}(|0\rangle-i|1\rangle)$. Qubit spaces can be composed using a tensor product, and we denote $|\phi \psi\rangle=|\phi\rangle \otimes|\psi\rangle$.

Hybrid quantum-classical systems include classical data, which can be represented in a qubit space as orthonormal basis vectors (e.g. by representing a logical 0 as the state $|0\rangle$ and a logical 1 as $|1\rangle$ ), but additionally include a trace or measurement operation, which probabilistically projects a qubit into a vector in an orthogonal basis. The resulting probabilistic distribution of pure states is called a mixed state, and is better represented by a density matrix, a positive semi-definite Hermitian operator of trace one in the $\left(\mathbb{C}^{2 \times 2}\right)^{\otimes n}$ Hilbert space, for an $n$-qubit system. Given a probabilistic distribution of pure states $\left\{\left(p_{i},\left|\phi_{i}\right\rangle\right)\right\}$, their density matrix is constructed as $\sum_{i} p_{i}\left|\phi_{i}\right\rangle\left\langle\phi_{i}\right|$, where $\langle\phi|=| \phi\rangle^{\dagger}$.

Quantum circuit diagrams consist of horizontal lines carrying each the information of one qubit, read from right to left, with some attached gates applying unitary transformations over the qubit states. We use the universal set of operations $\left\{\mathrm{CNOT}, \mathrm{X}_{\alpha}, \mathrm{Z}_{\alpha}, \mathrm{H}\right\}$ for pure-quantum diagrams. When $\alpha$ is limited to multiples of $\frac{\pi}{4}$ this roughly corresponds to the approximately universal Clifford $+\mathrm{T}$ group. Some rotation gates have specific names, such as $\mathrm{Z}=\mathrm{Z}_{\pi}$, $\mathrm{X}=\mathrm{X}_{\pi}, \mathrm{S}=\mathrm{Z}_{\frac{\pi}{2}}, \mathrm{HSH}=\mathrm{X}_{\frac{\pi}{2}}$, and $\mathrm{T}=\mathrm{Z}_{\frac{\pi}{4}}$. We additionally include ancilla 
initialization and termination, and swaps. The representation of each mentioned gate is reproduced here.

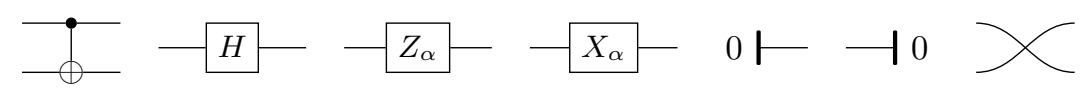

Hybrid circuits represent bit-carrying classical wires using doubled lines and extend the set of gates with some classical operations such as $\{$ NOT, XOR, AND , classical fan-out, bit swaps, measurement, qubit preparation, and classically controlled versions of the $\mathrm{X}_{\pi}$ and $\mathrm{Z}_{\pi}$ gates. We depict them respectively as follows.

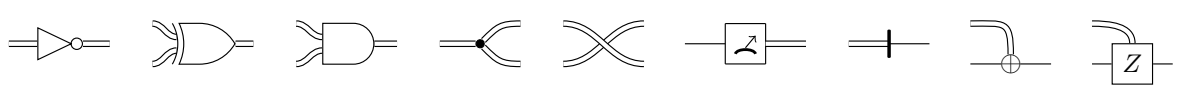

Circuits are inductively constructed from these generators, wire identities, and parallel and serial composition, ensuring that only wires of the same type connect with each other.

In this work we restrict the input to circuits with classical parity logic, choosing not to include AND gates due to the complexity of their representation as $\mathrm{ZX}_{\perp}$ diagrams, which might result in the introduction of additional non-Clifford gates during the extraction procedure (refer to Section 9 for further discussion).

The ZX-calculus is a formal graphical language which provides a fine-grained representation of quantum operations. We present a brief introduction to its definition, including the $\mathrm{ZX}_{\doteq}$ extension to represent classical operations. Refer to 24] for a complete description of both calculi.

ZX diagrams representing pure-quantum linear maps are composed by wires, spiders, and Hadamard boxes. We read the diagrams from right to left and represent inputs and outputs as open-ended wires. The Hadamard box $\square-$ swaps the computational and diagonal basis, mapping $|0\rangle$ to $|+\rangle,|1\rangle$ to $|-\rangle$ and vice versa. The spiders are arbitrary-degree nodes labelled with a real phase $\alpha \in[0,2 \pi)$ that come in either green or red color, named Z- and X-spiders respectively. When $\alpha$ is a multiple $\pi$ or $\frac{\pi}{2}$, we call them Pauli or Clifford-spiders respectively. We refer to the set of spiders connected to outputs and inputs of the diagram as $\mathrm{O}$ and I respectively, and call their members output- and inputspiders.

A degree-2 green (resp. red) spider corresponds to applying a $\mathrm{Z}_{\alpha}\left(\mathrm{X}_{\alpha}\right)$ operation over a qubit. Phaseless spiders represents nodes with phase 0 and can be interpreted as copying the computational basis vectors in the case of green spiders, or the diagonal basis vectors for red spiders.

$$
\alpha|00\rangle+\beta|11\rangle+\gamma|01\rangle+\theta|10\rangle>\bigcirc<\alpha|000\rangle+\beta|111\rangle
$$

Spiders of the same color can be fused together, adding their phases. It is important to note that the relative position of the nodes in ZX diagrams do not alter their interpretation, as only the topology matters. 
The ZX-calculus comes equipped with a complete set of formal rewrite rules [13]. We reproduce it here ignoring scalars.
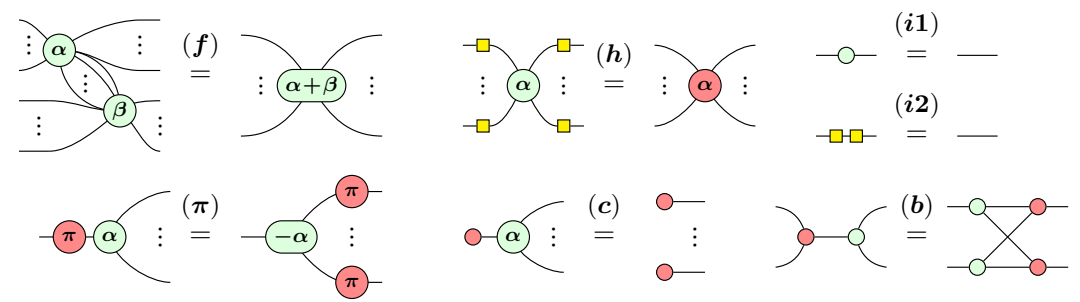

The $\mathrm{ZX}_{ \pm}$calculus 3 is an extension to the ZX-calculus which is able to easily describe interactions with the environment. The diagrams have a standard interpretation as completely positive linear maps between quantum mixed states (cf. Appendix A for a formal description). In addition to the ZX generators and rewrite rules, the calculus introduces a ground generator $(\stackrel{\perp}{\leftrightarrows})$ which represent the tracing operation, or the discarding of information. When connected to a degree-3 green spider, this can correspond to a measurement operation over the computational basis or a qubit initialization from a bit.

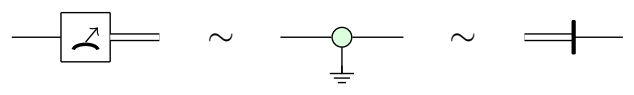

We refer to the spiders attached to $\doteq$ generators as $\doteq$-spiders. Notice that we use the same kind of wire for both classical and quantum data, since as previously discussed we can encode the latter as the former. We will later introduce a method to differentiate between the two types of wire by using the $\doteq$-spiders in Section 7

$\mathrm{ZX}_{\perp}$ extends the set of rewriting rules with the following additions.

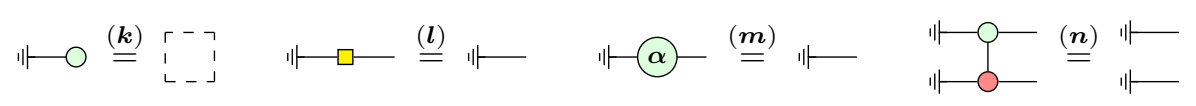

Intuitively, the $\doteq$ generator discards any operation applied over a single qubit. Multiple discards can be combined into one vio the following rule, derived from rules $(\boldsymbol{m}),(\boldsymbol{n})$, and $(\boldsymbol{k})$.

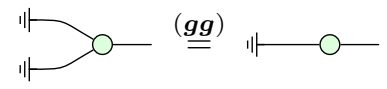

For simplicity in our diagrams, we replace solely as notation the Hadamard boxes with "Hadamard wires" drawn in blue, as follows.

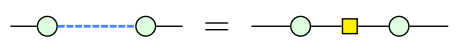

We introduce two additional derived equations. One to erase duplicated Hadamard wires, as proven by Duncan et al. 8], and another to discard them, from a combination of rules $(\boldsymbol{m})$ and $(\boldsymbol{l})$.

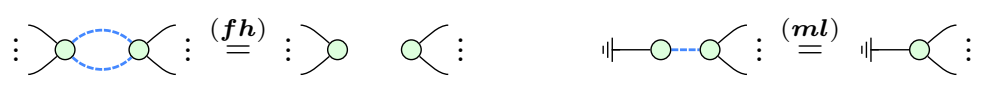


We utilise !-box notation [20] to represent infinite families of diagrams with segments that can be repeated 0 or more times. In the following sections it will be useful to use this notation for depicting more complex diagrams. Here we present an example of its usage.

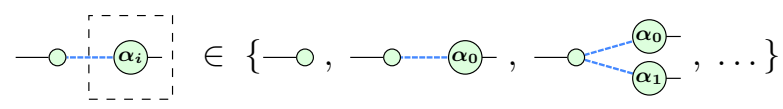

\section{Graph-like diagrams and focused gFlow}

A ZX diagram is said to be in graph-like form [8 when it contains only Zspiders connected by Hadamard wires, there are no parallel edges nor self-loops, and no spider is connected to more than one input or output. We define the graph-like form for $\mathrm{ZX}_{\doteq}$ diagrams and include a weaker version allowing a node to connect to an input, a ground, and any number of outputs simultaneously. When defining a translation from quantum circuits into $\mathrm{ZX}_{\overline{\bar{\Xi}}}$ diagrams it will be simpler to initially generate weakly graph-like diagrams and transform the final result into the strict version afterwards.

Definition 1. A $Z X_{\bar{亠}}$ diagram is graph-like (respectively weakly graph-like) when:

1. All spiders are Z-spiders.

2. Z-spiders are only connected via Hadamard edges.

3. There are no parallel Hadamard edges or self-loops.

4. There is no pair of connected $\doteq-$-spiders.

5. Every input, output, or $\doteq$ is connected to a Z-spider.

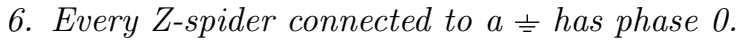

7. Every Z-spider is connected to at most one input, one output, or one $\doteq$ (at most one input and at most one $\doteq)$.

Proposition 1. Every $Z X_{\doteq}$ diagram is equivalent to a weakly graph-like $Z X_{\doteq}$ diagram. Indeed, Duncan et al. [8] proved that any pure-ZX diagram is equivalent to a graph-like one. The proof can be extended to weakly graph-like $Z X_{\perp}$ diagrams simply by applying rule (l) to eliminate Hadamards connected to $\doteq$ generators, rule $(\boldsymbol{g g})$ to eliminate duplicated $\doteq$ connected to the same spider, and rule (n) to disconnect wires between $\stackrel{-}{=}$-spiders.

Lemma 1. There exists an algorithm to transform an arbitrary $Z X_{\doteq}$ diagram into an equivalent strictly graph-like diagram.

Proof. By adding identity spiders to the inputs and outputs. Cf. Appendix B.

Once a diagram is in a weakly graph-like form, all its spiders as well as all its internal connections are of the same kind. We can refer to its underlying structure as a simple undirected graph, marking the nodes connected to inputs and outputs. In addition, $\doteq$ generators or the $\doteq$-spiders connected to them can be seen as outputs discarding information into the environment. This is known as the underlying open graph of a diagram. 
Definition 2. An open graph is a triple $(G, S, T)$ where $G=(V, E)$ is an undirected graph, and $S \subseteq V$ is a set of sources and $T \subseteq V$ is a set of sinks. For a weakly graph-like $Z X_{\perp}$ diagram $D$, the underlying open graph $G(D)$ is the open graph whose vertices are spiders $D$, whose edges correspond to Hadamard edges, whose set $S$ is the subset of spiders connected to the inputs of $D$, and whose set $T$ is the subset of spiders connected to the outputs of $D$ or to ground generators.

The underlying open graph of a ZX diagram produced from our translation of quantum circuits verify a graph-theoretic invariant called focused gFlow [19]. This structure - originally conceived for graph states in measurement based quantum computation - gives a notion of flow of information and time on the diagram. It will be required to guide the extraction strategy in Section 6 .

Definition 3. Given an open graph $G$, a focused gFlow $(g, \prec)$ on $G$ consists of a function $g: \bar{T} \rightarrow 2^{\bar{S}}$ and a partial order $\prec$ on the vertices $V$ of $G$ such that for all $u \in \bar{T}, \operatorname{Odd}_{G}(g(u)) \cap \bar{T}=\{u\}$ and $\forall v \in g(u), u \prec v$ where $2^{\bar{S}}$ is the powerset of $\bar{S}$ and $\operatorname{Odd}_{G}(A):=\{v \in V(G)|| N(v) \cap A \mid \equiv 1 \bmod 2\}$ is the odd neighbourhood of $A$.

\section{Translation of hybrid quantum-classical circuits}

We describe our translation from hybrid quantum-classical circuits into strictly graph-like $\mathrm{ZX}_{\doteq}$ diagrams by steps. First, we translate each individual gate directly into a weakly graph-like diagram and connect them with regular wires. We define this translation $T(\cdot)$ by inductively translating the gates as described in Table 1 immediately followed by the application of the spider fusion rule $(\boldsymbol{f})$ and rules $(\boldsymbol{g g})$ and $(\boldsymbol{f} \boldsymbol{h})$ to remove all regular wires, duplicated $\doteq$ generators, and parallel Hadamard wires, ensuring that the final combined diagram is in a weakly graph-like form. An example of this translation is shown in Figure1.

Notice that the translation maps both classical and quantum wires to regular $\mathrm{ZX}_{\perp}$ diagram edges. We keep track of which inputs and outputs of the diagram were connected to classical wires and introduce $\doteq$ generators for the operations that interact with the environment. In Section 6 we present a method to detect the sections of the final circuit that can be implemented as classical operations by looking at the classical inputs/outputs and the $\doteq$ generators, independently of which wires where originally classical.

Lemma 2. The $Z X_{\perp}$ diagram resulting from the translation $T(\cdot)$ is weakly graphlike.

Proof. By induction on the circuit construction. Cf. Appendix B.

After the translation, we can apply Lemma 1 to obtain a strictly graph-like diagram. This step essentially separates the $\doteq$ generators from the inputs and outputs, allowing the optimization procedure to move them around and let them interact with other parts of the diagram. 
A. Borgna et al.

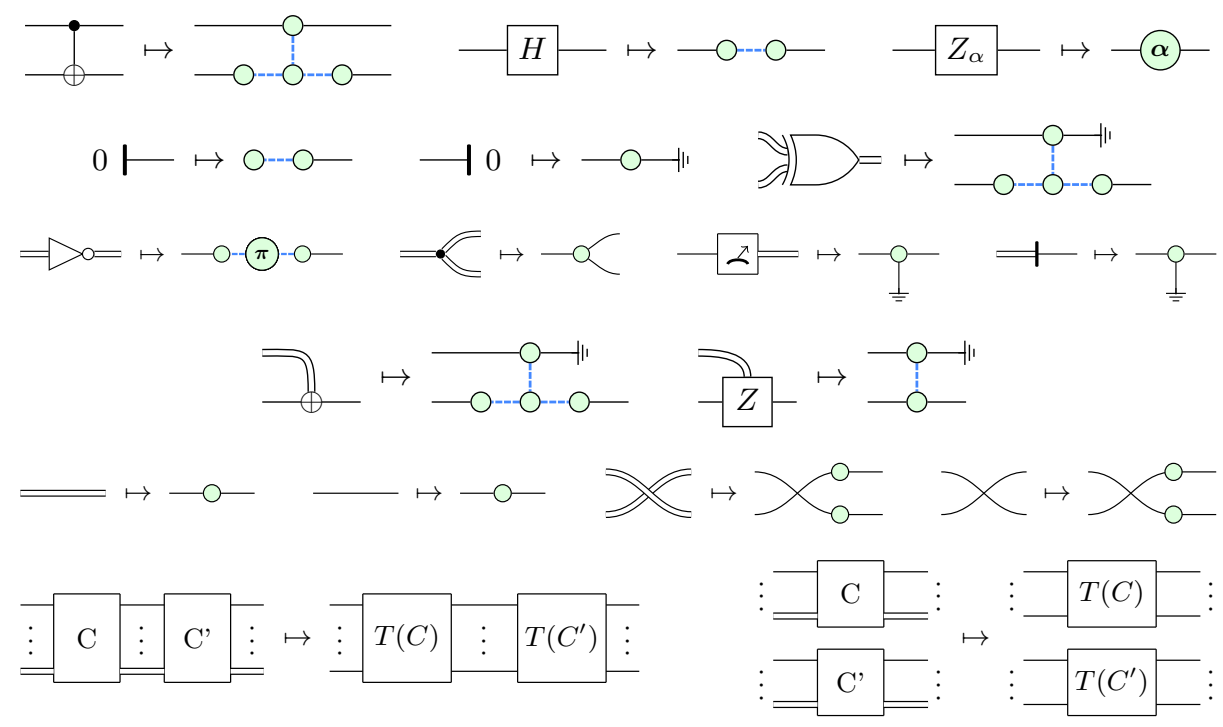

Table 1: Translation from hybrid quantum-classical circuits into $\mathrm{ZX}_{\doteq}$ diagrams.
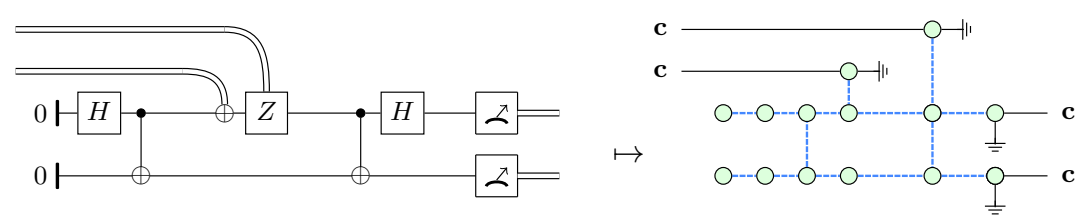

Figure 1: Example translation of the superdense coding circuit into a $\mathrm{ZX}_{\doteq}$ diagram with labelled inputs and outputs, and subsequent application of the spider-fusion rule.

Lemma 3. If $C$ is a hybrid quantum-classical circuit and $D$ is the graph-like $Z X_{\perp}$ diagram obtained from the translation $T(C)$ and Lemma 1 , then $G(D)$ admits a focused gFlow.

Proof. By induction on C. Cf. Appendix B.

\section{Grounded ZX optimization}

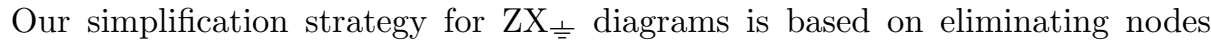
from the diagram by systematically applying a number of rewriting rules while preserving the existence of a focused gFlow. In this section we introduce the new rules, define a strategy to maximize their effectiveness, and finally use it together with the pure-ZX optimization to define our algorithm. 


\subsection{Basic simplification rules}

Duncan et al. 8] presented the following gFlow-preserving local complementation and pivoting rules for the ZX calculus in their optimization procedure. These rules effectively reduce the size of the diagram by at least one node on each application by eliminating internal proper-Clifford spiders and Pauli spider pairs respectively.

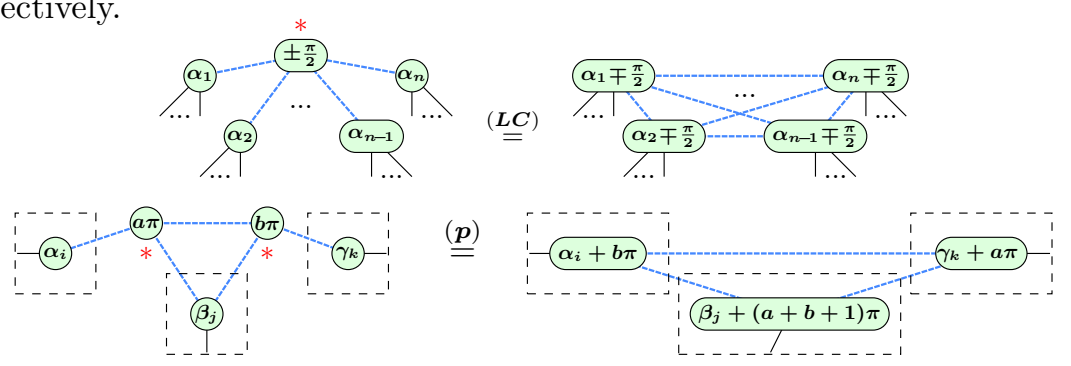

These rules can be applied directly in $\mathrm{ZX}_{\doteq}$ diagrams when the target spiders are not connected to $\mathrm{a} \doteq$ generator. For the cases where some of the target spiders are $\doteq$-spiders, we introduce the following altered rules. Their derivation can be found in Appendix $\mathrm{C}$.

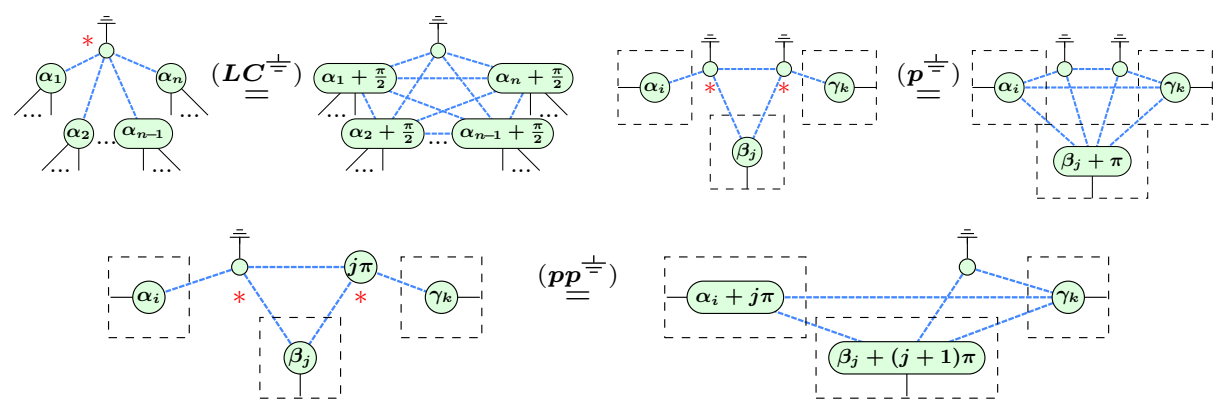

Notice that both rules $\left(\boldsymbol{L} \boldsymbol{C}^{\doteq}\right)$ and $\left(\boldsymbol{p}^{\doteq}\right)$ do not decrease the number of spiders in the diagram. As such, we will focus on rule $\left(\boldsymbol{p} \boldsymbol{p}^{\stackrel{\perp}{\Xi}}\right)$ for our optimization.

If $\left(\boldsymbol{p} \boldsymbol{p}^{\stackrel{\doteq}{\Xi}}\right)$ is applied with a non- $=$ spider connected to a boundary, the rule produces a $\doteq$-spider connected to an input or output thus needing to add an identity operation as described in Lemma 1 to preserve the graph-like property. Since in this case we add additional nodes to the graph, we will only apply rule $\left(\boldsymbol{p} \boldsymbol{p}^{\stackrel{\perp}{=}}\right)$ on a boundary spider if it can be followed by another node-removing rule.

Additionally, we will use rules $(\boldsymbol{m l})$ and $(\boldsymbol{k})$ directly to remove nodes in the diagram when there are $\doteq$-spiders with degree 1 or 0 in the graph, respectively.

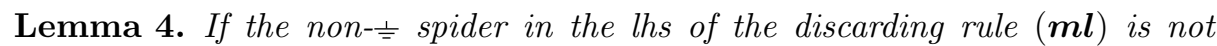
connected to an output or input, then applying the rule over a graph-like diagram $D$ preserves the existence of a focused gFlow.

Proof. If the non- $\stackrel{\perp}{=}$ spider in the lhs is not connected to an input or output of the diagram, then applying the rule does not break the graph-like property of $D$. The preservation of the gFlow follows from $\doteq$-spiders being sinks of the underlying open graph. 


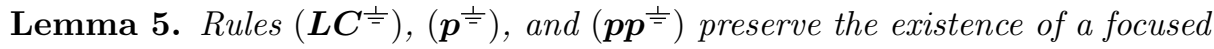
gFlow.

Proof. Notice that that rules $\left(\boldsymbol{L} \boldsymbol{C}^{\doteq}\right),\left(\boldsymbol{p}^{\doteq}\right)$, and $\left(\boldsymbol{p} \boldsymbol{p}^{\stackrel{\perp}{\Xi}}\right)$ are compositions of gFlow-preserving rules.

\subsection{Ground-cut simplification}

The previously introduced rewriting rules require a simplification strategy to apply them. A simple solution is to try to find a match for each rule and apply them iteratively until no more matches are available. We describe a strategy that can find additional rule matches by operating on the biadjacency matrix

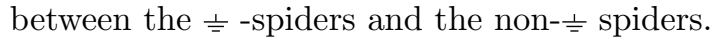

Definition 4. The ground-cut of a graph-like $Z X_{\perp}$ diagram $D$ is the cut resulting from splitting the $\doteq$ and non- $\doteq$ spiders in $G(D)$.

Since the diagram is graph-like, there are no internal wires in the $\doteq$ partition. Given a $Z_{\beth}$ diagram $D$, we denote $M_{D}$ the biadjacency matrix of its groundcut, where rows correspond to $\doteq$-spiders and columns correspond to non- $\doteq$ spiders. We can apply all elementary row operations on the matrix by rewriting the diagram. The addition operation between the rows corresponding to the $\stackrel{\perp}{=}$ -spider $u$ and the $\stackrel{\perp}{\bar{\prime}}$-spider $v$ can be implemented via the following rule, the derivation of which can be found in Appendix C.

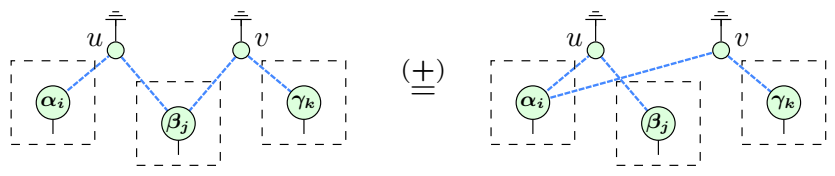

Using the elementary row operations we can apply Gaussian elimination on the ground-cut biadjacency matrix of a graph-like $\mathrm{ZX}_{\overline{\bar{\Sigma}}}$ diagram, generating in the process an equivalent diagram whose ground-cut biadjacency matrix is in reduced echelon form.

Any row in the ground-cut biadjacency matrix left without non-zero elements

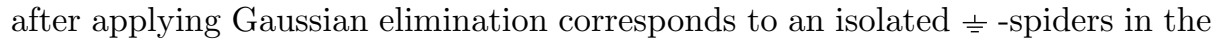
diagram that can be eliminated by rule $(\boldsymbol{k})$. If the reduced row echelon form of the biadjacency matrix contains a row with exactly one non-zero elements, then that element corresponds to an isolated $\triangleq$-spider and non- $\doteq$ spider pair in the diagram and therefore we can apply rule $(\boldsymbol{m l})$ to remove the non- $\doteq$ spider.

\subsection{The Algorithm}

Based on the previous strategy, we define a terminating procedure which turns any graph-like $\mathrm{ZX} \mathrm{X}_{\doteq}$ diagram into an equivalent simplified diagram that cannot be further reduced.

Definition 5. A graph-like $Z X_{\perp}$ diagram is in simplified-form if it does not contain any of the following, up to single-qubit unitaries on the inputs and outputs. 


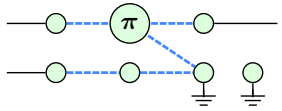

(a) Diagram not in simplified-form

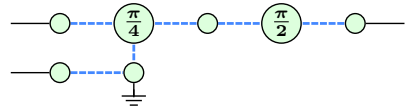

(b) Diagram in simplified-form

Figure 2: Example of simplified and non-simplified diagrams.

- Interior proper Clifford spiders.

- Adjacent pairs of interior Pauli spiders.

- Interior Pauli spiders adjacent to boundary spiders.

- Interior Pauli spiders adjacent to $\doteq$-spiders.

- Degree-1 $\doteq$-spiders not connected to input or output spiders.

- Connected components not containing inputs nor outputs.

An example of diagrams satisfying and not satisfying this property is shown in Figure 2

We define an optimization algorithm that produces diagrams in simplifiedform by piggybacking on the pure optimization procedure. This optimization applies the local complementations $(\boldsymbol{L} \boldsymbol{C})$ and pivoting $(\boldsymbol{p})$ rules until there are no interior proper Clifford spiders or adjacent pairs of non- $\stackrel{\doteq}{=}$ interior Pauli spiders. After the initial pure simplification, we continue our optimization as follows.

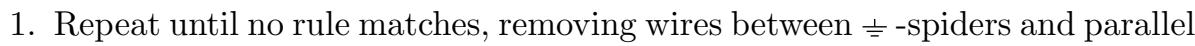
Hadamard connections after each step:

(a) Run Gaussian elimination on the ground-cut of the diagram as described in Section 5.2

(b) Remove the grounds corresponding to null rows with rule $(\boldsymbol{k})$.

(c) If any row of the biadjacency matrix has a single non-zero element, corresponding to $\mathrm{a} \doteq-$-spider connected to a spider $v$, then:

i. If $v$ is not a boundary spider, apply rule $(\boldsymbol{m l})$.

ii. If $v$ is a boundary spider and $v$ is adjacent to a Pauli spider, apply rule $(\boldsymbol{m l})$ immediately followed by the procedure from Lemma 1 to make the diagram graph-like again. Then delete the Pauli neighbour using rule $\left(\boldsymbol{p} \boldsymbol{p}^{\bar{\doteq}}\right)$, to ensure that the step removes at least one node.

(d) Apply Pauli spider elimination rule ( $\left.\boldsymbol{p} \boldsymbol{p}^{\stackrel{\perp}{=}}\right)$ until there are no Pauli spiders connected to ground spiders.

2. Remove any connected component of the graph without inputs or outputs.

Notice that each cycle the loop reduces the number of nodes in the graph, so this is a terminating procedure. Additionally, since each applied rule preserves the existence of a gFlow the final diagram admits a gFlow. An example run of the algorithm is shown in Figure 3. 


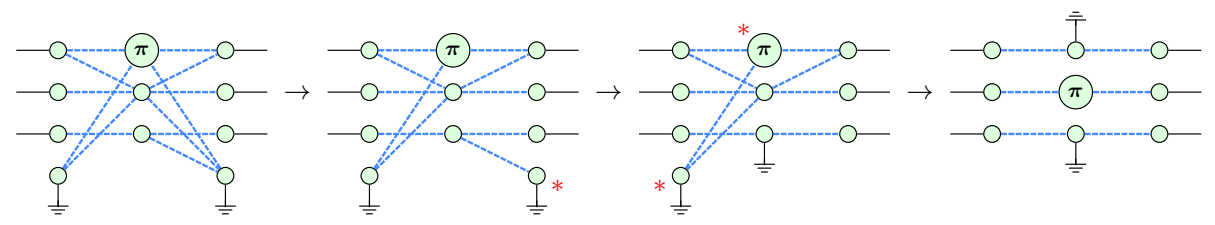

Figure 3: Example of a diagram optimization applying a ground-cut simplification, a discard rule, and a Pauli elimination.

\section{Circuit extraction}

Here we describe a general circuit extraction procedure for graph-like $\mathrm{ZX}_{\doteq}$ diagrams admitting a focused gFlow into hybrid quantum-classical circuits, by modifying the procedure for pure diagrams from the Clifford optimization. We present the pseudocode in Algorithm 1 and an example of an execution in Appendix D.

The algorithm progresses through the diagram from right-to-left, maintaining a set of spiders F, called the frontier, which represents the unextracted spiders connected to the extracted segment. Each frontier spider is assigned an output qubit line $\mathrm{Q}(v)$. This set is initially populated by the nodes connected to outputs of the diagram. The strategy is to proceed backwards by steps, adding unextracted spiders into the frontier and deleting some of them to extract operations on the output circuit, in back-to-front order.

To find candidate spiders to add to the frontier we apply Gaussian elimination on the biadjacency matrix of the frontier and non-frontier spiders, similarly to the optimization method described in Section 5.2. The gFlow property of the graph ensures that we can always progress by extracting a node. It suffices to look at the set of non-frontier vertices maximal in the order and notice that, after

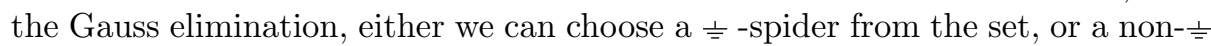
spider that has a single connection to the frontier. A careful implementation of the biadjacency matrix row and column ordering can reduce the number of $\doteq$-spider extractions when no non- $\doteq$ candidates are available. We require the following proposition to apply the row additions on the graph (Duncan et al. [8], Proposition 7.1).

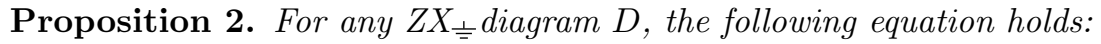

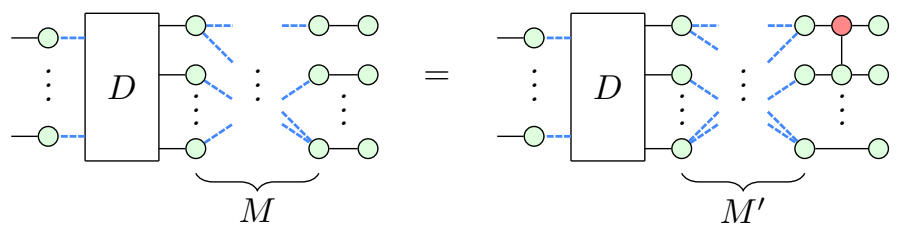

where $M$ describes the biadjacency matrix of the relevant vertices, and $M^{\prime}$ is the matrix produced by adding row 2 to row 1 in $M$. Furthermore, if the diagram on the LHS has a focused gFlow, then so does the RHS. 


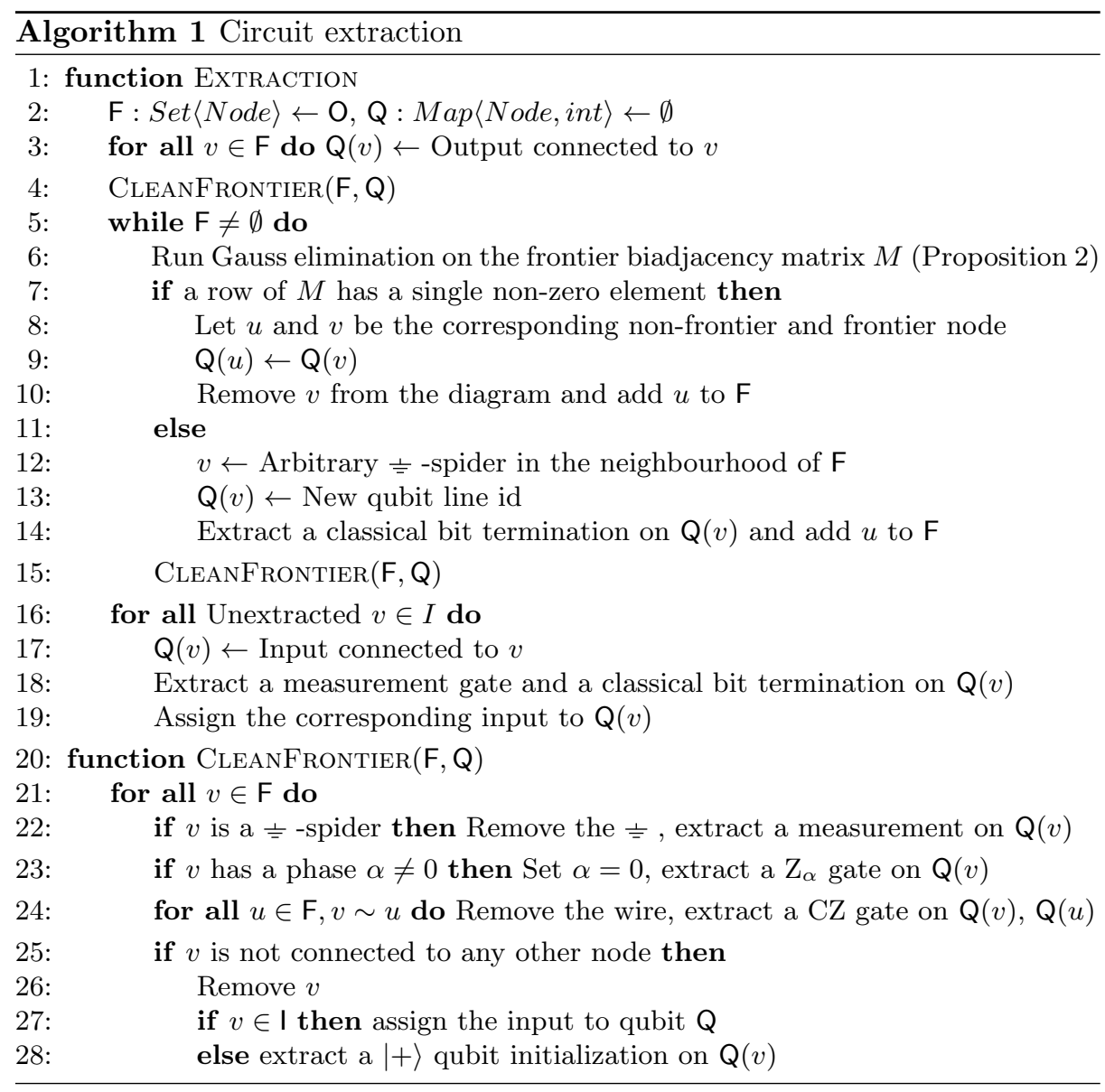

In our pseudocode, the call to CLEANFrontiER ensures that $F$ only contains phaseless spiders without internal wires. Notice that it preserves the gFlow since it only modifies edges between sink nodes, and removes spiders with no other connections. After the while loop terminates, all outputs of the circuit will have been extracted. If there are inputs left unextracted, and since the diagram had a gFlow, we can discard them directly via measurement operations.

Finally, we add any necessary swap operations to map the inputs to the correct lines, and insert qubit initializations and measurements at inputs and outputs marked as classical. In Section 7 we detail a method to better detect the internal parts of the circuit that can be implemented classically.

In any case, each step of the while loop in Algorithm 1 line 5, preserves the gFlow of the diagram, and we can show that it terminates in at most $|V|$ steps: Indeed, if there are no non-frontier spiders, then a call to CLEANFronTIER will remove all nodes from the frontier. Moreover, each step of the while loop in line 5 moves one non-frontier spider to F. 


\section{Circuit classicalization}

The extraction procedure described in Section 6 produces correct circuits that are almost completely composed by quantum gates and wires, without any classical operation. In this section we describe the general problem of detecting parts of the circuits that can be realized as classical operations, and introduce an efficient heuristic solution based on a local-search. Notice, however, that while we aim to recognize all classically realizable operations in the circuit the characteristics of each quantum computer may dictate the final choice between quantum and classical operators by taking into account the costs of exchanging data between both realms.

Given a $Z_{\doteq}$ diagram, we decorate its wires using the set of labels $\mathcal{L}=$ $\{\mathrm{Q}, \mathrm{X}, \mathrm{Y}, \mathrm{Z}, \perp\}$. The label $\mathrm{Z}$ means that this particular wire can be replaced by a classical wire (possibly precomposed with a standard basis measurement and postcomposed by a qubit initialisation in the standard basis depending on whether the connected wires are also classical or not), and similarly for $\mathrm{X}$ and $\mathrm{Y}$ by adapting the basis of measurement/initialisation to the diagonal or $Y$ basis. $\mathrm{Q}$ means that the wire is a quantum wire, and finally $\perp$ means that the wire can be removed by precomposing with $\mathrm{a} \doteq$ and postcomposing with a maximally mixed state. The set of labels form a partial order, $\mathrm{Q} \geq \mathrm{X}, \mathrm{Y}, \mathrm{Z} \geq \perp$.

A labelling $L$ of a diagram $D$ is a map from its edges into a pair of labels. The two labels, drawn at each end of the wire, indicate the origin of the constraint. Intuitively, $\frac{\mathrm{z} \quad A}{A}$ means that the wire is produced in such a way that guarantees that the qubit carries classical information encoded in the computational basis, whereas $\stackrel{A}{2}$ means that the wire can be replaced by a classical wire because some process will force this qubit to be in that basis - for instance, it is going to be measured in the standard basis and thus one can already measure this qubit in the standard basis and use a classical wire-. We define a partial order between labellings of a diagram as the natural lift from the partial order of the labels.

Each label corresponds to a density matrix subspace of $\mathbb{C}^{2 \times 2}$, representing all possible mixed states allowed by that particular kind of wire.

$$
\begin{array}{ll}
\mathrm{Q}=\mathbb{C}^{2 \times 2} & \mathrm{Z}=\left\{\alpha|0\rangle\langle 0|+\beta| 0\rangle\left\langle 0|| \alpha, \beta \in \mathbb{R}_{\geq 0}, \alpha+\beta=1\right\}\right. \\
\perp=\left\{\frac{1}{2}|0\rangle\left\langle 0\left|+\frac{1}{2}\right| 1\right\rangle\langle 1|\right\} & \mathrm{X}=\left\{\alpha|+\rangle\langle+|+\beta|-\rangle-|| \alpha, \beta \in \mathbb{R}_{\geq 0}, \alpha+\beta=1\right\} \\
& \mathrm{Y}=\left\{\alpha|\circlearrowright\rangle\langle\circlearrowright|+\beta| \circlearrowleft\rangle\left\langle|| \alpha, \beta \in \mathbb{R}_{\geq 0}, \alpha+\beta=1\right\}\right.
\end{array}
$$

Notice that the greatest common ancestor $A \sqcup B$ corresponds to the intersection of the sets.

Intuitively, a labelling is valid if we can cut any wire in the diagram and, after forcing a valid state in the inputs and outputs, we get a valid state in the cut terminals. That is, we rearrange the diagram to transform all outputs into inputs and connect the cut terminals as outputs, as shown on the right. Then, applying an arbitrary input $\rho \in\left(\bigotimes_{i}^{n} A_{i}\right) \otimes\left(\bigotimes_{j}^{m} D_{j}\right)$ to the diagrams produces a result in $E \otimes F$. 
Notice that if $A$ is a valid label for a wire then any $B \geq A$ is also valid, and in particular $Q$ is always a valid label. We can then omit unnecessary labels in the diagrams, marking them implicitly as Q.

Given a $\mathrm{ZX}_{\doteq}$ diagram $D$ with marked classical inputs and outputs, we define the classicalization problem as finding a minimal valid labeling where the inputs and outputs are labelled as $\mathrm{Q}$ or $\mathrm{Z}$ accordingly.

\subsection{Local-search algorithm}

We present a local-search labelling procedure for $\mathrm{ZX}_{ \pm}$diagrams with explicit Hadamard gates - replacing the Hadamard wires - and only green spiders, that produces locally minimal labellings by propagating the labels over individual spiders. A diagram resulting from the circuit extraction in Section 6 can be transformed to have only green spiders by applying the color-change rule $(\boldsymbol{h})$. This restriction is purely for simplicity in our definition, as the equivalent functions can be defined easily for red spiders.

We introduce a number of operations over the labels. First, a binary function representing the result of combining two wires via a phaseless green spider, $\star: \mathcal{L} \times \mathcal{L} \rightarrow \mathcal{L}$.

$$
\begin{array}{lllll}
\mathrm{Z} \star A=Z & \mathrm{X} \star A=A & \mathrm{Y} \star \mathrm{Y}=\mathrm{X} & \mathrm{Q} \star \mathrm{Y}=\mathrm{Q} & \perp \star \mathrm{Y}=\perp \\
A \star \mathrm{Z}=Z & A \star \mathrm{X}=A & \mathrm{Y} \star \mathrm{Q}=\mathrm{Q} & \mathrm{Q} \star \mathrm{Q}=\mathrm{Q} & \perp \star \mathrm{Q}=\mathrm{Z} \\
& & \mathrm{Y} \star \perp=\perp & \mathrm{Q} \star \perp=\mathrm{Z} & \perp \star \perp=\perp
\end{array}
$$

Notice that $(\mathcal{L}, \star)$ is a commutative monoid with $\mathrm{X}$ as neutral element. We also define a "Z rotation" operation for $\alpha \in[0,2 \pi), \operatorname{rot}_{\alpha}: \mathcal{L} \rightarrow \mathcal{L}$.

$$
\begin{aligned}
& \operatorname{rot}_{\alpha}(\mathrm{Z})=\mathrm{Z} \quad \operatorname{rot}_{\alpha}(\mathrm{Q})=\mathrm{Q} \quad \operatorname{rot}_{\alpha}(\perp)=\perp \\
& \operatorname{rot}_{\alpha}(X)=\left\{\begin{array}{ll}
X & \text { if } \alpha \in\{0, \pi\} \\
Y & \text { if } \alpha \in\left\{\frac{1}{2} \pi, \frac{3}{4} \pi\right\} \\
Q & \text { otherwise }
\end{array} \quad \operatorname{rot}_{\alpha}(Y)= \begin{cases}Y & \text { if } \alpha \in\{0, \pi\} \\
X & \text { if } \alpha \in\left\{\frac{1}{2} \pi, \frac{3}{4} \pi\right\} \\
Q & \text { otherwise }\end{cases} \right.
\end{aligned}
$$

This corresponds to the identity if $\alpha \in\{0, \pi\}$, and in general $\operatorname{rot}_{\alpha}(A) \star b \geq$ $\operatorname{rot}_{\alpha}(A \star B)$.

Finally, we define a function $\mathrm{H}$ representing the application of the Hadamard operation over a label, $\mathrm{H}: \mathcal{L} \rightarrow \mathcal{L}$.

$$
\mathrm{H}(\mathrm{Q})=\mathrm{Q} \quad \mathrm{H}(\mathrm{X})=\mathrm{Z} \quad \mathrm{H}(\mathrm{Z})=\mathrm{X} \quad \mathrm{H}(\mathrm{Y})=\mathrm{Y} \quad \mathrm{H}(\perp)=\perp
$$

Our classical detection procedure starts by labelling any classical input or output with a $\mathrm{Z}$ label, and any $\doteq$ with $\mathrm{a} \perp$ label, and the rest of the diagram wires with $Q$.

It then proceeds by propagating the labels using the following rules:

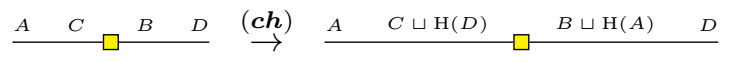




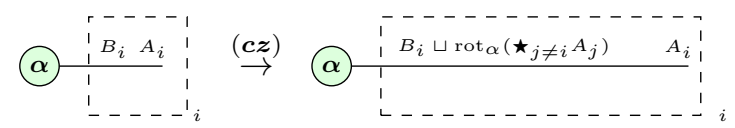

For any labels $A, B, C, D, E, F \in \mathcal{L}$.

We apply these rules until there are no more labels to change. Since each time we replace labels with lesser ones in the order, the procedure terminates. Finally, we can interpret wires with a classical label in any direction as classically realisable. We show an example of a labeled diagram in Figure 4

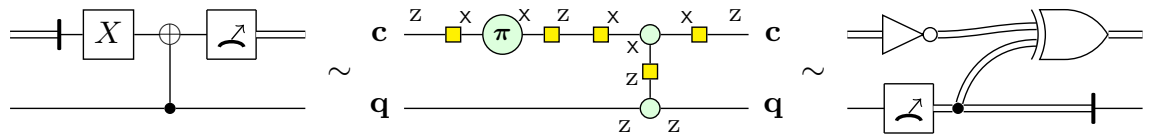

Figure 4: Example of a local-search classicalization. Q labels are omitted.

Lemma 6. The local-search labelling algorithm produces a valid labeling according to the standard interpretation of the $Z X_{\doteq}$ calculus.

Proof. By proving that both rules $(\boldsymbol{c h})$ and $(\boldsymbol{c z})$ produce valid labellings, cf. Appendix B.

Remark 1. The local-search labelling does not always reach a globally minimum labeling. Consider for example the following input diagram.

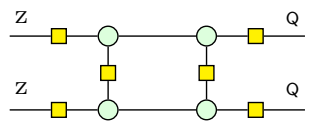

Here, although the diagram is equivalent to the identity and the outputs could theoretically be labelled as Z, the entangled wires in the middle do not allow for local propagation of the classical labels past the double controlled $\mathrm{Z}$ gates.

\section{Implementation}

We have implemented each of the algorithms presented in this work as an extension to the open source Python library PyZX [16] by modifying its implementation of $\mathrm{ZX}$ diagrams to admit $\mathrm{ZX}_{\doteq}$ primitives. A repository with the code is available at http://github.com/aborgna/pyzx/tree/zxgnd. We additionally implemented a naïve $\mathrm{ZX}_{ \pm}$extension of the pure Clifford optimization for comparison purposes, which doesn't use any of our $\cong$ rewriting rules. When applied to pure quantum circuits, our algorithm does not perform additional optimizations after the Clifford procedure and therefore achieves the same benchmark results recorded by Duncan et al. on the circuit set described by Amy et al. 11.

We tested the procedure over two classes of randomly generated circuits, and measured the size of the resulting diagram as the number of spiders left after the 
optimization. This metric correlates with the size of the final circuit, although the algorithmic noise caused by the arbitrary choices in the extraction procedure may result in some cases in bigger extracted circuit after a reduction step.

The first test generates Clifford $+\mathrm{T}$ circuits with measurements by applying randomly chosen gates from the set $\{\mathrm{CNOT}, \mathrm{S}, \mathrm{HSH}, \mathrm{T}, \mathrm{Meas}$ over a fixed number of qubits, where Meas are measurement gates on a qubit immediately followed by a qubit initialization. We fix the probability of choosing a CNOT, $\mathrm{S}$, or HSH gate to 0.2 each and vary the probabilities for $\mathrm{T}$ and Meas in the remaining 0.4 . These circuits present a general worst case, where there is no additional classical structure to exploit during the hybrid circuit optimization.

The second type of generated operations are classical parity-logic circuits. These consist on a number of classical inputs, fixed at 10, where we apply randomly chosen operations from the set $\{$ NOT, XOR, Fanout $\}$ with probabilities $0.3,0.3$, and 0.4 respectively.

In Figure 5 we compare the results of our optimization using the Clifford optimization as baseline. Figure $5 \mathrm{a}$ shows the reduction of diagram size when running the algorithm on randomly generated Clifford $+\mathrm{T}$ circuits with measurement. We vary the probability of generating a measurement gate between 0 and 0.2 while correspondingly changing the probability of generating a T-gate between 0.4 and 0.2 , and show the results for different combinations of qubit and gate quantities. We remark that the optimization produces noticeably smaller diagrams once enough $\nRightarrow$ generators start interacting with each other. There is a critical threshold of measurement gate probability, specially visible in the cases with 8 qubits and 1024 gates, where with high probability the outputs of the diagram become disconnected from the inputs due to the $\doteq$ interactions. This results in our algorithm optimizing the circuits to produce a constant result while discarding their input.

Figure $5 \mathrm{~b}$ shows the comparison of diagram size between our procedure and the Clifford optimization when run over classical parity circuits. The optimization produces consistently smaller diagrams, generally achieving the theoretical minimal number of $\doteq$ generators, equal to the number of inputs. We further remark that in all of the tested cases the classicalization procedure was able to detect that all the extracted operations on the optimized parity-logic circuit were classically realisable.

The runtime of our algorithm implementation is polynomial in the size of the circuit. As with the Clifford optimization, the cost of our optimization and extraction processes is dominated by the Gauss elimination steps. For the groundnode rewriting rules, our unoptimized implementation is roughly $\mathcal{O}\left(n^{2} * k^{2}\right)$ in the worst case with $k$ being the number of measurement gates and $n$ the number of gates, but in practice it behaves cubically on the number of gates due to the sparseness of the diagrams. The implementation was not developed with a focus on the runtime cost, and some possible optimizations may reduce this bound. 

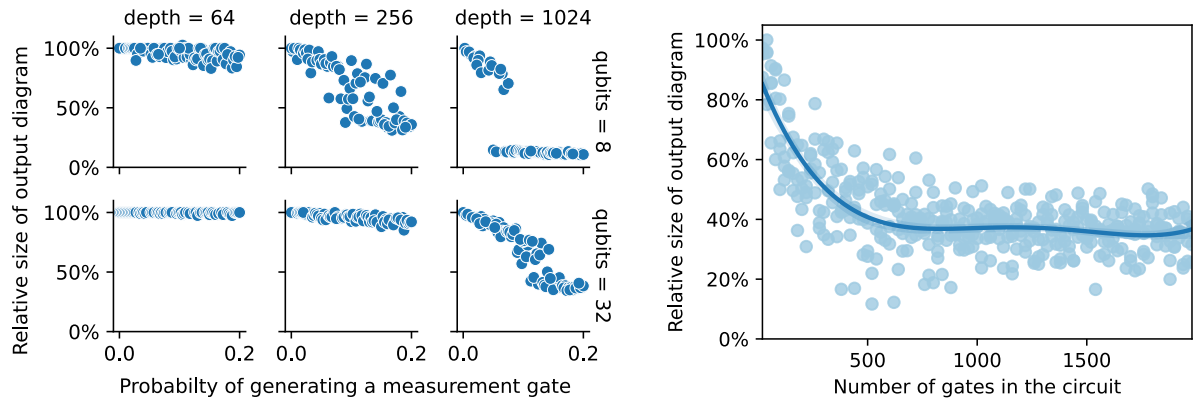

(a) Diagram size reduction on Clifford+T (b) Diagram size reduction on parity-logic circuits with measurements. circuits.

Figure 5: Benchmark results on randomly generated diagrams.

\section{Discussion and future work}

We introduced an optimization procedure for optimizing hybrid classical circuits inspired by previous work on pure circuit optimization using the ZX calculus. The process is composed by a translation step, the optimization of the diagrams, an extraction back into circuits and finally a detection of classically-realisable operations. Our translation operation produces diagrams which admit a focused gFlow, a property that we maintain during the optimization and require during the extraction. For our optimization step we defined a series of rewrite strategies to reduce the size of the diagrams, and introduced a strategy to find additional optimization opportunities by applying Gaussian elimination on the biadjacency matrix of the ground-cut of the diagram. Our extraction procedure initially generates circuits without classical operations. Hence, we introduced a classicalization heuristic for arbitrary circuits that is able to replace quantum operations by their classical equivalent, where possible.

Kissinger and van de Wetering [17] defined a procedure based on the Clifford optimization to reduce the T-gate count in quantum circuits by defining new structures in the graphs called phase gadgets and operating over their phases. Their work can be easily extended to $\mathrm{ZX}_{\doteq}$, where the $\doteq$ generators act as an absorbing element for the gadgets phases. However, rules such as (+) prove to be strictly more powerful than applying the pure phase gadget rules over $\stackrel{-}{=}$ gadgets. In general, the phase-gadget optimization affects an independent section of the structure of the diagram compared to ours, and can be applied with it.

During our definition of the optimization process we decided to restrict the input circuits to parity classical logic, excluding AND and OR gates. This does not raise from an inherent limitation of the system but from a practical standpoint. The $\mathrm{ZX}_{\perp}$ calculus is able to represent AND operations in what equates to the Clifford $+\mathrm{T}$ decomposition of the Toffoli gate, introducing multiple T-gates and CNOT gates to the circuit 22]. The multiple spiders would be dispersed around the diagram during the optimization step, potentially breaking the pat- 
tern formed by the AND gate and replacing it with multiple quantum operations. This can produce the unexpected result of introducing expensive quantum operations in an originally pure classical circuit. A possible next step for this work would be to use an alternative diagrammatic representation better adapted to represent arbitrary boolean circuit such as the $\mathrm{ZH}$ calculus.

Acknowledgements The authors would like to thank Kostia Chardonnet and Renaud Vilmart for their suggestions on the classicalization problem, and John van de Wetering for his help with the pyzx library. This work was supported in part by the French National Research Agency (ANR) under the research project SoftQPRO ANR-17-CE250009-02, and by the DGE of the French Ministry of Industry under the research project PIA-GDN/QuantEx P163746-484124, and by the project STIC-AmSud project Qapla' 21-SITC-10.

\section{Bibliography}

[1] Amy, M., Maslov, D., Mosca, M.: Polynomial-time T-depth optimization of Clifford $+\mathrm{T}$ circuits via matroid partitioning. IEEE T. Comp. Aid. D. 33(10), 1476-1489 (2014).

[2] de Beaudrap, N., Horsman, D.: The ZX calculus is a language for surface code lattice surgery. Quantum 4, 218 (2020).

[3] Carette, T., Jeandel, E., et al.: Completeness of graphical languages for mixed states quantum mechanics. In: Proc. ICALP. pp. 108:1-108:15 (2019).

[4] Chancellor, N., Kissinger, et al.: Graphical structures for design and verification of quantum error correction (2016),

[5] Coecke, B., Duncan, R.: Interacting quantum observables. In: Proc. ICALP. pp. 298-310 (2008).

[6] Coeke, B., Kissinger, A.: Picturing Quantum Processes. (2017)

[7] Cross, A.W., Bishop, L.S., et al.: Open quantum assembly language (2017).

[8] Duncan, R., Kissinger, et al.: Graph-theoretic simplification of quantum circuits with the zx-calculus. Quantum 4, 279 (2020).

[9] Duncan, R., Lucas, M.: Verifying the Steane code with Quantomatic. In: Proc. QPL. pp. 33-49 (2013).

[10] Duncan, R., Perdrix, S.: Rewriting measurement-based quantum computations with generalised flow. In: Proc. ICALP. pp. 285-296 (2010).

[11] Green, A.S., Lumsdaine, et al.: Quipper: a scalable quantum programming language. In: Proc. PLDI. pp. 333-342 (2013).

[12] Heyfron, L., Campbell, E.T.: An efficient quantum compiler that reduces T count. (2018)

[13] Jeandel, E., Perdrix, S., Vilmart, R.: Completeness of the ZX-calculus. LMCS, 16(2), pp.11-1 (2020).

[14] Jozsa, R.: An introduction to measurement based quantum computation. NATO Science Series, III, vol. 199, pp. 137-158 (2005).

[15] Khammassi, N., Guerreschi, et al.: cqasm v1.0: Towards a common quantum assembly language (2018). 
[16] Kissinger, A., van de Wetering, J.: PyZX: Large scale automated diagrammatic reasoning. ENTCS 318, 229-241 (2020).

[17] Kissinger, A., van de Wetering, J.: Reducing T-count with the ZX-calculus. (2020)

[18] Li, G., Zhou, L., Yu, et al.: Projection-based runtime assertions for testing and debugging quantum programs. PAPL4(OOPSLA) (Nov 2020).

[19] Mhalla, M., Murao, et al. Which graph states are useful for quantum information processing? In: Proc. TQC. pp. 174-187 (2014).

[20] Miller-Bakewell, H.: Finite verification of infinite families of diagram equations. In: Proc. QPL. pp. 27-52 (2019).

[21] Selinger, P.: Dagger compact closed categories and completely positive maps: (ext. abst.). In: Proc. QPL. pp. 139-163. (2005).

[22] Selinger, P.: Quantum circuits of T-depth one. Ph. Rev. A 87(4) (2013).

[23] Steiger, D.S., Häner, T., Troyer, M.: ProjectQ: an open source software framework for quantum computing. Quantum 2, 49 (2018).

[24] van de Wetering, J.: ZX-calculus for the working quantum computer scientist. (2020)

[25] Zhou, H., Byrd, G.T.: Quantum circuits for dynamic runtime assertions in quantum computation. IEEE Comp, Arch. Lett. 18(2), 111-114 (2019). 


\section{A Semantics of the ZX-ground calculus}

Carette et al. 3] define an interpretation of $\mathrm{ZX}_{\Perp}$ - diagrams using a CPM construction (cf. Reference 21]). We describe it here without the categorical language, as an interpretation of diagrams into density matrices and completely positive maps modulo scalars.

Let $\mathcal{D}_{n} \subseteq \mathbb{C}^{2^{n} \times 2^{n}}$ be the set of n-qubit density matrices. There exist a functor $\llbracket \cdot \rrbracket: \mathrm{ZX}_{\perp} \rightarrow \mathbf{C P M}($ Qubit) which associates to any diagram $D: n \rightarrow m$ a completely positive map $\llbracket D \rrbracket: \mathcal{D}_{n} \rightarrow \mathcal{D}_{m}$, inductively defined as follows.

$$
\begin{aligned}
& \llbracket D_{1} \otimes D_{2} \rrbracket:=\llbracket D_{1} \rrbracket \otimes \llbracket D_{2} \rrbracket \quad \llbracket D_{2} \circ D_{1} \rrbracket:=\llbracket D_{2} \rrbracket \circ \llbracket D_{1} \rrbracket \quad \llbracket\left[\begin{array}{c}
c \\
\vdots \\
-
\end{array}\right]:=(1) \quad \llbracket @ \rrbracket:=\left(1+e^{2 i \alpha}\right) \\
& \llbracket — \rrbracket:=i d \quad \llbracket>\rrbracket:=\rho \mapsto U \rho U^{\dagger} \text { where } U=|00 \times 00|+|01 \times 10|+|10 \times 01|+|11 \times 11| \\
& \llbracket \square \square \rrbracket:=\rho \mapsto H \rho H^{\dagger} \text { where } H=\frac{1}{\sqrt{2}}\left(\begin{array}{cc}
1 & 1 \\
1 & -1
\end{array}\right) \\
& \llbracket \Vdash \rrbracket:=|0\rangle\langle 0|+| 1\rangle\langle 1| \quad \llbracket \longrightarrow-1 \rrbracket:=\rho \mapsto\langle 0|\rho| 0\rangle+\langle 1|\rho| 1\rangle \\
& \llbracket n @ \vdots \rrbracket:=\rho \mapsto A \rho A^{\dagger} \text { if } n+m>0 \text {, where } A=\left|0^{m} \times 0^{n}\right|+e^{i \alpha}\left|1^{m} \times 1^{n}\right| \\
& \llbracket n \vdots \propto \vdots m \rrbracket:=\llbracket-\square-\rrbracket^{\otimes m} \circ \llbracket n \vdots \propto\left(m \rrbracket \circ \llbracket-\square-\rrbracket^{\otimes n}\right.
\end{aligned}
$$

From the compositional rules, we can see that a ground attached to a green spider corresponds to a measurement over the computational basis.

$$
\left.\llbracket n \vdots \vdots m:=\rho \mapsto\left|0^{m}\right\rangle\left\langle 0^{n}|\rho| 0^{n}\right\rangle 0^{m}|+| 1^{m}\right\rangle\left\langle 1^{n}|\rho| 1^{n}\right\rangle\left\langle 1^{m}\right|
$$

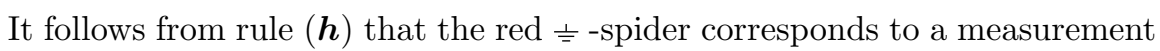
over the diagonal basis:

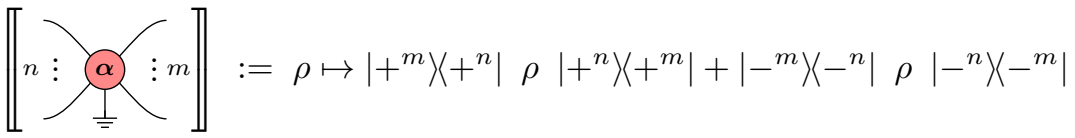

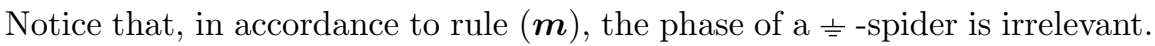

\section{B Extended proofs}

\section{B.1 Proofs of section 3}

Lemma (1). There exists an algorithm to transform an arbitrary $Z X_{\doteq}$ diagram into an equivalent strictly graph-like diagram. 
Proof. The strict graph-like condition limits to 1 the number of input, output, and ground generators connected to each spider, in addition to the weakly graphlike restrictions. By Proposition 11, the diagram can be rewritten into an equivalent weakly graph-like diagram $D$. We describe an algorithm which modifies $D$ to comply with the additional restriction.

For each spider $v$ in $D$ connected to at least two inputs, outputs or $\doteq$ generators, add two Z-spiders and Hadamard wires to each connected input and output as follows.

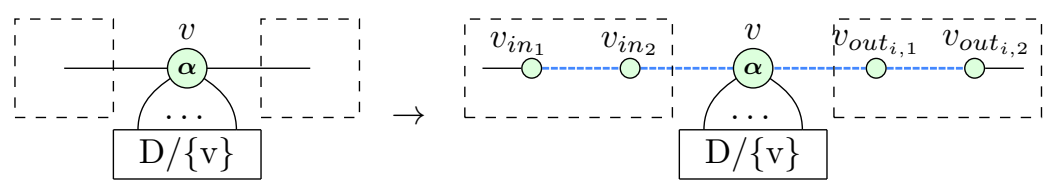

Notice that $v$ may have at most one connected input. The introduced spiders correspond to identity operations, and therefore the procedure generates a strictly graph-like diagram equivalent to $D$.

\section{B.2 Proofs of section 4}

Lemma (2). The $Z X_{\perp}$-diagram resulting from the translation $T(\cdot)$ is weakly graph-like.

Proof. Notice that all the translation rules aside from the serial composition generate weakly graph-like diagrams trivially.

For the serial composition, notice that both $T(C)$ and $T\left(C^{\prime}\right)$ are weakly graph-like by the induction hypothesis and therefore all spiders in the resulting diagram are Z-spiders, all inputs and outputs are connected to Z-spiders, no two inputs are connected to the same spider, and all spiders connected to $\doteq$ -generators have phase 0 . The internal edges added by the composition will therefore connect two green spiders, which will be merged by the fusion rule application.

The fusion step may create spiders connected to two ground generators, one of which is removed by the application of rule $(\boldsymbol{g g})$. It may also generate parallel Hadamard wires, which are removed by the application of rule $(\boldsymbol{f h})$.

Therefore, the translation generates weakly graph-like $\mathrm{ZX}_{\Perp}$-diagrams.

For the proof of Lemma 3, we use a weaker version of the focused gFlow invariant called causal flow 19].

Definition 6. Given an open graph $G$, a causal flow $(f, \prec)$ on $G$ consists of a function $f: \bar{T} \rightarrow \bar{S}$ and a partial order $\prec$ on the set $V$ satisfying the following properties:
1. $f(v) \sim v$
2. $v \prec f(v)$
3. If $u \sim f(v)$ and $u \neq v$ then $v \prec u$ 
where $u \sim v$ if $u$ is connected to $v$ in the graph, and $\bar{A}=V(G) / A$.

Duncan et al. [8, Theorem B.3] prove the following lemma relating both flow properties:

Proposition 3. If $G$ admits a causal flow, then there exists a valid focused gFlow for $G$.

Lemma 7. If $D$ is a weakly graph-like $Z X_{\perp}$ diagram and $G(D)$ admits a causal flow, the strictly graph-like diagram generated by the algorithm described in Lemma 1 admits a causal flow.

Proof. Let $\left(f_{D}, \prec_{D}\right)$ be a valid causal flow for $D$ and let $D^{\prime}$ be the resulting diagram after applying rule 1 over a node $v$. We construct a causal flow for $D^{\prime}$ by defining a function $f_{D^{\prime}}$ and relation $\prec D_{D^{\prime}}$ as the minimal objects such that

$-f_{D^{\prime}} \supseteq f_{D}$ and $\prec_{D^{\prime}} \supseteq \prec_{D}$.

- If $v$ is connected to an input in $D, f_{D^{\prime}}\left(v_{i n_{1}}\right)=v_{i n_{2}}, f_{D^{\prime}}\left(v_{i n_{2}}\right)=v$, and $\left(v_{i n_{1}}, v_{i n_{2}}\right),\left(v_{i n_{2}}, v\right) \in \prec_{D^{\prime}}$.

- If $v$ is connected to at least one output in $D$ and $v$ is not a $\doteq$-spider, $f_{D^{\prime}}(v)=v_{\text {out }}$.

- For each output $i$ connected to $v$ in $D,\left(v, v_{\text {out }_{i, 1}}\right),\left(v_{\text {out }_{i, 1}}, v_{\text {out }_{i, 2}}\right) \in \prec_{D^{\prime}}$ and $f_{D^{\prime}}\left(v_{\text {out }_{i, 1}}\right)=v_{\text {out }_{i, 2}}$.

Notice that $\left(f_{D^{\prime}}, \prec_{D^{\prime}}^{+}\right)$is a valid focused flow for $D^{\prime}$, where $\prec_{D^{\prime}}^{+}$is the transitive closure of $\prec_{D^{\prime}}$. Since the rule 1 preserves the focused flow, the resulting diagram after successive application admits a causal flow.

Lemma (3). If $C$ is a hybrid quantum-classical circuit and $D$ is the graphlike $Z X_{\perp}$-diagram obtained from the translation $T(C)$ and Lemma 1, then $G(D)$ admits a focused gFlow.

Proof. By Proposition 3 , it suffices to prove that $G(D)$ admits a causal flow. We proceed by induction on the construction of $C$.

- Notice that the translation of each base constructors cannot be further simplified by rules $(\boldsymbol{f}),(\boldsymbol{g g})$, or $(\boldsymbol{f} \boldsymbol{h})$, and the underlying open graph trivially admits a causal flow.

- If $C=C_{1} \otimes C_{2}$, let $D_{i}=T\left(C_{i}\right)$. Since the two circuits are not connected after the composition, they do not interact via the rules $(\boldsymbol{f}),(\boldsymbol{g g})$, or $(\boldsymbol{f} \boldsymbol{h})$, and therefore $D=D_{1} \otimes D_{2}$. By the induction hypothesis $G\left(D_{1}\right)$ and $G\left(D_{2}\right)$ admit some causal flow $\left(f_{1}, \prec_{1}\right)$ and $\left(f_{2}, \prec_{2}\right)$ respectively. Then, $\left(f_{1} \cup f_{2}, \prec_{1} \cup \prec_{2}\right)$ is a causal flow for $G(D)$.

- If $C=C_{1} \otimes C_{2}$, let $D_{i}=T\left(C_{i}\right)$. By the induction hypothesis $G\left(D_{1}\right)$ and $G\left(D_{2}\right)$ admit some causal flow $\left(f_{1}, \prec_{1}\right)$ and $\left(f_{2}, \prec_{2}\right)$ respectively. Notice that rule $(\boldsymbol{f})$ will be applied between each output of $C_{1}$ and the connected inputs in $C_{2}$. Let $f_{1}^{\prime}$ be a function and $\prec_{1}^{\prime}$ a relation such that for each vertex $v$ in $G\left(D_{1}\right)$ and corresponding non-empty set of inputs $\left\{u_{1}, \ldots, u_{k}\right\}$ in $G\left(D_{2}\right)$, $\forall v^{\prime}$ st. $f_{1}\left(v^{\prime}\right)=v, f_{1}^{\prime}\left(v^{\prime}\right)=u_{1}$ and $\forall v^{\prime} s t . v^{\prime} \prec_{1} v,\left\{\left(v^{\prime}, u_{i}\right)\right\}_{i=1}^{k} \subseteq \prec_{1}^{\prime}$. Notice 
that since $D_{2}$ is weakly graph-like, $u_{1}$ has exactly one corresponding sink node in $G\left(D_{1}\right)$. Additionally, for all $v_{1}, v_{2}$ non-output nodes in $G\left(D_{1}\right)$ let $f_{1}^{\prime}\left(v_{1}\right)=v_{2}$ if $f_{1}\left(v_{1}\right)=v_{2}$ and let $v_{1} \prec_{1}^{\prime} v_{2}$ if $v_{1} \prec_{1} v_{2}$. Then, $\left(f_{1}^{\prime} \cup f_{2},\left(\prec_{1}^{\prime}\right.\right.$ $\left.\cup \prec_{2}\right)^{+}$) is a causal flow for $G(D)$.

Then, by Lemma , the application of Lemma 1 preserves the existence of a causal flow.

\section{B.3 Proofs of section 6}

Lemma (??). Each step of the while loop in Algorithm 1. line 5, preserves the gFlow of the diagram.

Proof. By Proposition 2 the Gauss elimination application preserves the gFlow. Then, look at the set of non-frontier spiders maximal in the gFlow order.

If the set contains a non- $\triangleq$ spider $u$ then by definition $g(u) \in \mathrm{F}$. If $\operatorname{Odd}_{G}(g(u))$ does not contain $\doteq$ nodes, after the Gauss elimination there must be a frontier spider $v$ such that it is only connected to $u$. Therefore, removing $v$ and making $u$ a sink of the diagram does not break the gFlow.

On the other case, since $\stackrel{\perp}{=}$-spiders are always sinks of the diagram promoting them to the frontier does not modify the gFlow of the diagram.

Finally, the call to CleanFrontier does not modify the gFlow.

\section{B.4 Proofs of Section 7}

For the following proofs we use the interpretation of $\mathrm{ZX}_{\doteq}$ diagrams defined in Appendix A

Lemma 8. The labelling rule (ch) preserves the validity of the labelling.

Proof. We prove the validity of the replacement of label $B$. The replacement of label $C$ is the symmetric case.

Since the starting diagram has a valid labelling, $B \sqcup \mathrm{H}(A)$ is a valid label if $\mathrm{H}(A)$ is a valid label. Therefore the label is valid if $\forall \rho \in A \otimes D$,

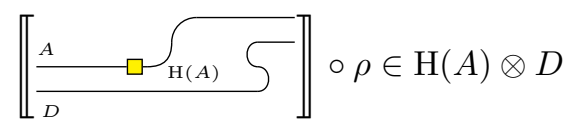

Notice that this is equivalent to requiring $\forall a \in A,\left(\mathrm{H} a \mathrm{H}^{\dagger}\right) \in \mathrm{H}(A)$, which follows from the definition of $\mathrm{H}(\cdot)$.

Lemma 9. The labelling rule (cz) preserves the validity of the labelling.

Proof. By induction on the number of wires.

- If $n=0$, there are no labelled wires. 
- If $n=1$, since the starting diagram has a valid labelling, $B_{1} \sqcup \operatorname{rot}_{\alpha}(\mathrm{X})$ is a valid labelling if $\operatorname{rot}_{\alpha}(\mathrm{X})$ is valid. Notice that

$$
\llbracket \propto-\rrbracket=\left(\begin{array}{cc}
1 & e^{-i \alpha} \\
e^{i \alpha} & 1
\end{array}\right) \in \operatorname{rot}_{\alpha}(\mathbf{X})
$$

- If $n=2$, we prove the validity of the replacement of label $B_{2}$. The replacement of label $B_{1}$ is the symmetric case.

Since the starting diagram has a valid labelling, $B_{2} \sqcup \operatorname{rot}_{\alpha}\left(A_{1}\right)$ is a valid label if $\operatorname{rot}_{\alpha}\left(A_{1}\right)$ is a valid label. Therefore the label is valid if $\forall \rho \in A_{1} \otimes B_{1}$,

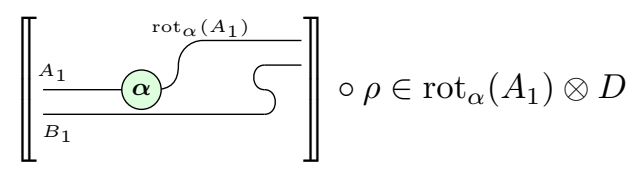

Notice that this is equivalent to requiring $\forall a \in A_{1},\left(U a U^{\dagger}\right) \in \operatorname{rot}_{\alpha}\left(A_{1}\right)$ for $U=|0\rangle\left\langle 0\left|+e^{i \alpha}\right| 1\right\rangle\langle 1|$, which follows from the definition of $\operatorname{rot}_{\alpha}(\cdot)$.

- If $n=3$, we prove the validity of the replacement of label $B_{1}$. The replacement of labels $B_{2}$ and $B_{3}$ are the symmetric case.

Since the starting diagram has a valid labelling, $B_{1} \sqcup \operatorname{rot}_{\alpha}\left(A_{2} \star A_{3}\right)$ is a valid label if $\operatorname{rot}_{\alpha}\left(A_{2} \star A_{3}\right)$ is a valid label. We can split the diagram as follows, adding an intermediary label.

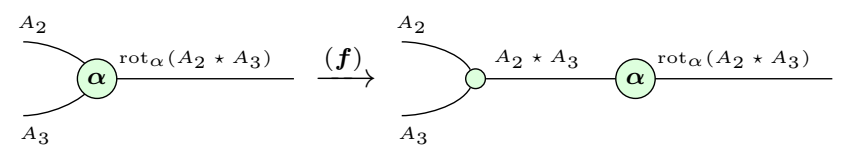

Notice that by the inductive hypothesis, the labelling step for the degree-2 spider is correct. Therefore it suffices to prove that the intermediary label $A_{2} \star A_{3}$ is valid, that is $\forall \rho \in A_{2} \star A_{3} \otimes \mathrm{Q}$,

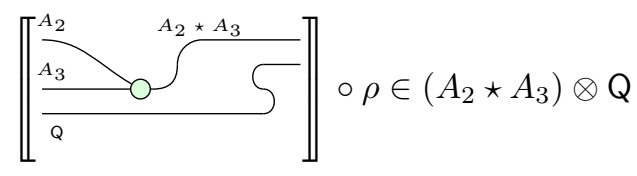

Notice that this is equivalent to requiring $\forall a_{2} \in A_{2}, a_{3} \in A_{3},\left(U\left(a_{2} \otimes\right.\right.$ $\left.\left.a_{3}\right) U^{\dagger}\right) \in A_{2} \star A_{3}$ for $U=|0\rangle\langle 00|+| 1\rangle\langle 11|$, which follows from the definition of $\star$.

- If $n>3$, for each $i$ and for some $k \neq i$ we can rewrite the diagram as follows, and apply $(\boldsymbol{c z})$ twice to produce the target $B_{i} \sqcup \operatorname{rot}_{\alpha}\left(\star_{j \neq i} A_{j}\right)$ label.

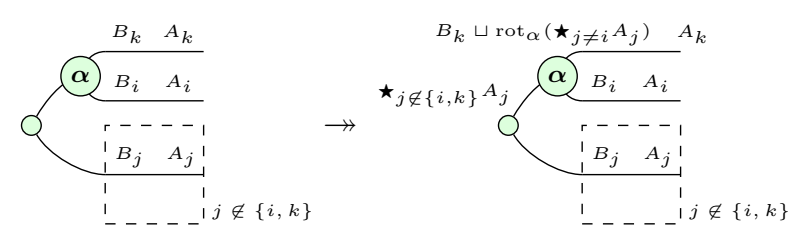

By inductive hypothesis, both rule applications produce valid labellings. 
Lemma (6). The local-search labelling algorithm produces a valid labeling according to the standard interpretation of the $Z X_{\doteq}$ calculus.

Proof. Notice that labelling every wire as $Q$ is always valid and hence the algorithm starts with a valid labelling. By Lemmas 8 and 9 , each step applying rules $(\boldsymbol{c z})$ and $(\boldsymbol{c h})$ preserve the validity and therefore the final labelling is valid.

\section{Rule derivations}

Here we present the derivation of rules presented in Section 5 .

- Rule $\left(\boldsymbol{L} \boldsymbol{C}^{\doteq}\right)$ :

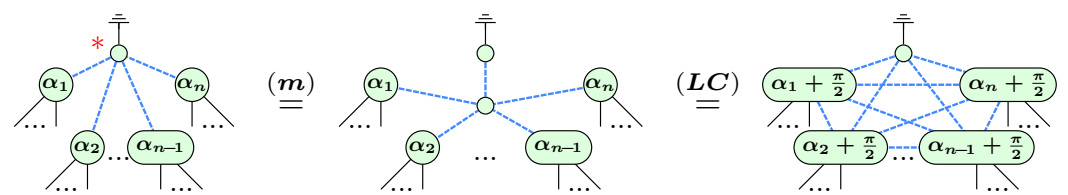

$-\operatorname{Rule}\left(\boldsymbol{p}^{\stackrel{\perp}{\bar{\Xi}}}\right)$ :
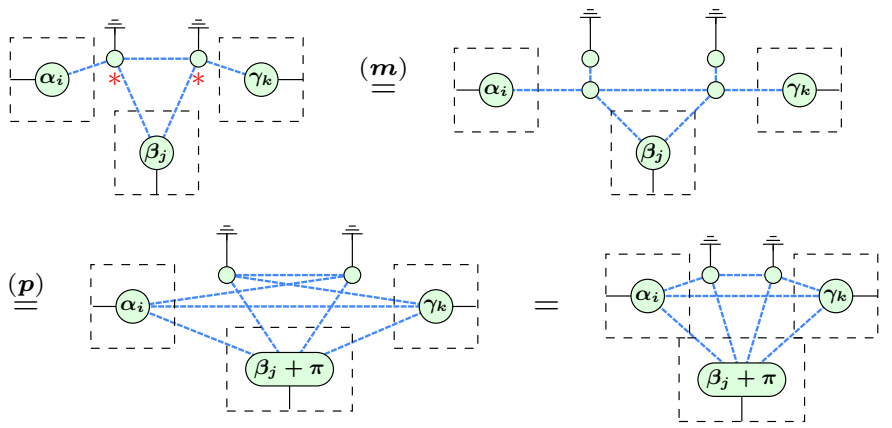

- Rule $\left(\boldsymbol{p} \boldsymbol{p}^{\doteq}\right)$ :

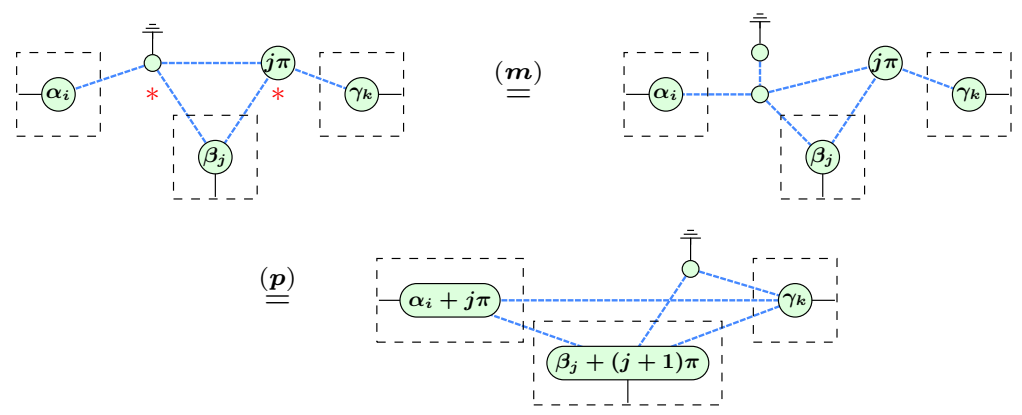

- Rule (+):

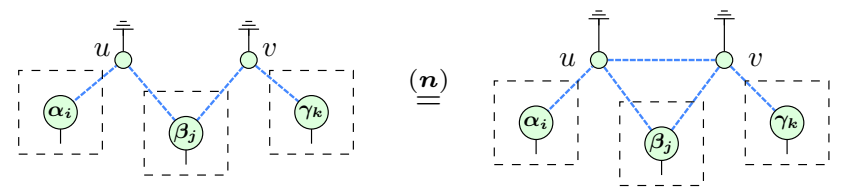




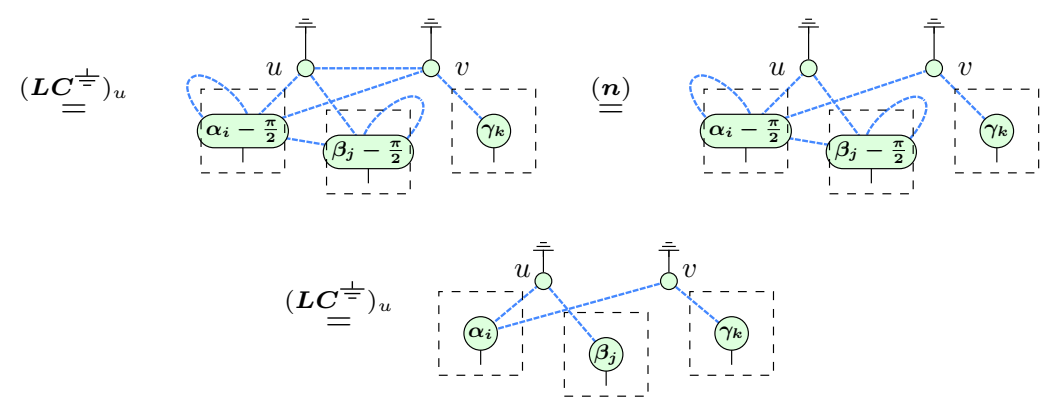

\section{Extraction examples}

We show here an example of running the extraction procedure on a diagram. The frontier set is demarcated with a dashed red box, and the extracted circuit is represented with their ZX equivalent directly connected to the right of the frontier.

We start with the frontier initialized as the output vertices, and directly extract any $\stackrel{\perp}{=}$.

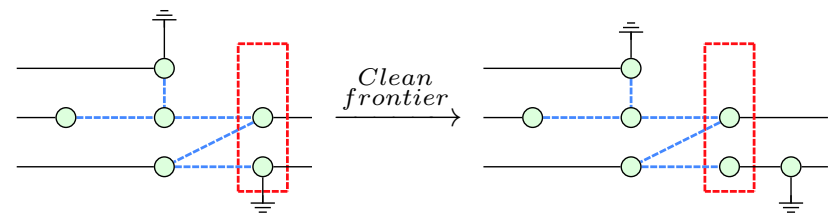

During each step of the algorithm, a maximal non-extracted element in the gFlow order is chosen. Candidates can be chosen efficiently without calculating the gFlow by running Gauss elimination on the biadjacency matrix between the border and the non-extracted spiders, and mirroring the row-sum operations using a gFlow preserving rewrite rule on the diagram.

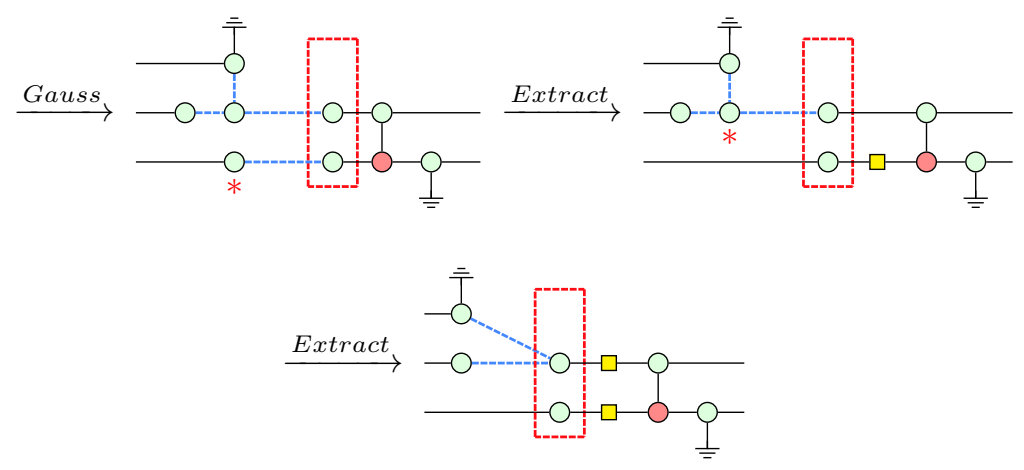

Now, there are no candidate frontier spiders connected to a single unextracted

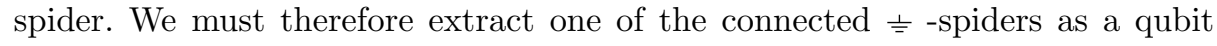


termination.
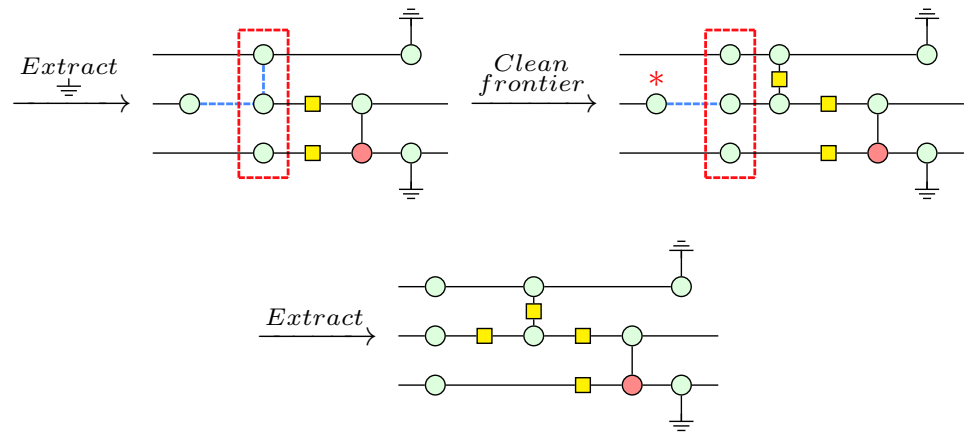

If after any step there are nodes in the frontier that are not connected to any internal spider then they can be removed from the frontier and extracted as a qubit initialization, as show in the following example.

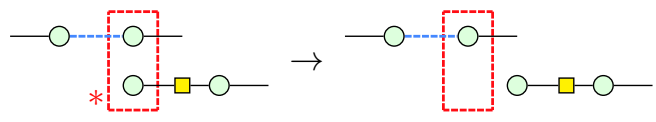

